III.

\title{
Carcinoma of the Fallopian Tubes, Right Ovary and Appendix.
}

\author{
By Walter Tate, M.D., F.R.C.P.
}

M.T., æt. 44, married, was admitted to St. Thomas's Hospital November 30; discharged December 26, 1908. She had always had good health, had been married 24 years, but had had no pregnancy. For 18 months she had noticed that her periods had become very scanty, and this condition persisted until three months before admission, when a large clot was passed from the vagina; this was unaccompanied by pain. Immediately following the passage of the clot the patient lost a considerable amount of bright red blood, and there was slight continuous loss up to the time of admission with exacerbations about once a fortnight which the patient regarded as periods. During these 3 months the patient had lost weight, but had pain in the back and left hip and had had three febrile attacks which were looked upon as being due to influenza. She was anæmic.

On examination of the abdomen nothing abnormal could be found, and the heart and lungs appeared to be normal. There was no trace of albumin in the urine.

On vaginal examination a hard mass was felt filling the right side of the pelvis. The surface of the mass was irregular, and several knobs could be felt on its surface bulging the vaginal fornix. The mass was adherent to the lateral pelvic wall and appeared to push the uterus over to the left side. The uterus was very difficult to define. Per rectum the hard mass was very easily felt and was bulging backwards in the right half of the pelvis.

Operation (December 4). On opening the abdomen the Fallopian tubes were found to be affected and bound down by adhesions to the back of the uterus; the right tube was thickened, the left distended. The adhesions round the tubes and ovaries were broken down and both appendages ligatured and removed: the appendix was adherent to the right appendages and was also excised. The omentum showed a large number of small growths, and the part containing these was removed. Bleeding points were secured, the pelvis sponged out and the abdomen closed. The patient made an uninterrupted recovery, and left hospital on the 23rd day after operation.

The parts removed consisted of the Fallopian tubes and ovaries, the vermiform appendix and a piece of omentum containing small deposits of growth. The right ovary was solid and brawny in 
consistence, and was about the size of a pigeon's egg; the right tube was very tortuous, its external surface was covered with adhesions and it was firmly adherent to the ovary. The wall was thickened and contained plaques of growth, the interior was filled with caseous material, the mucous membrane had disappeared. The whole tube was about as thick as the little finger. The left tube was expanded to the size of a large hen's egg, the surface was covered with adhesions; the wall contained plaques of growth and in other places was quite thin. The contents were similar to the fluid material found in dermoid cysts, the mucous membrane had disappeared. The left ovary appeared normal. The appendix was enlarged at its tip to about the size of a marble, the rest appeared healthy. The piece of the great omentum contained small masses of growth about the size of millet seeds. The parts removed were submitted to microscopical examination and the Fallopian tubes, right ovary and appendix showed columnar-celled carcinoma.

Although there was a carcinomatous growth of the vermiform appendix, it seems right to regard this case as primary carcinoma of the Fallopian tube, seeing that there was disease present in both tubes, the carcinoma on the more advanced side having spread and involved the appendix. For the abstract of this case I am indebted to Dr. J. P. Hedley.

IV.

\title{
A Case of Malignant Papilloma of the Fallopian Tube.
}

\author{
By T. P. LeGG, M.S. (Lond.), F.R.C.S., \\ Surgeon to the Royal Free Hospital, W.C.
}

THE patient, aged 42, was first seen on November 7, 1906. She complained of pain in the abdomen and vaginal discharge. She stated that 2 years and 4 months ago sharp colicky pains began to be felt in the left iliac and lumbar regions, and were accompanied by a blood-stained vaginal discharge. The pain had increased in severity and the discharge in amount, so that for the last two months before coming for advice she had been obliged to give up work. The pain was relieved in the recumbent position, but the increasing size of the abdomen caused much discomfort.

Menstruation had been quite regular, and there had been no increase in the amount lost. Unfortunately the notes do not say how many times the patient has been pregnant. Except for the present illness she appeared to have been quite healthy. 